\title{
Fourier spectra of radially periodic images with a non-symmetric radial period
}

\author{
Isaac Amidror \\ Laboratoire de Systèmes Périphériques (LSP), Ecole Polytechnique Fédérale de Lausanne \\ (EPFL), 1015 Lausanne, Switzerland
}

Received 1 January 1999, in final form 21 June 1999

\begin{abstract}
It has been previously shown that a radially periodic image having a symmetric radial period can be decomposed into a circular Fourier series of circular cosine functions with radial frequencies of $f=1 / T, 2 / T, \ldots$, and that its Fourier spectrum consists of a series of half-order derivative impulse rings with radii $f=n / T$ (which are the Fourier transforms of the circular cosines in the sum). In the present paper these results are extended to the general case of radially periodic images, where the radial period does not necessarily have a symmetric profile. Such a general radially periodic function can be decomposed into a circular Fourier series which is a weighted sum of circular cosine and sine functions with radial frequencies of $f=1 / T, 2 / T, \ldots$ In terms of the spectral domain, the Fourier spectrum of a general radially periodic function consists of half-order derivative impulse rings with radii $f=n / T$ (which are the Fourier transforms of the weighted circular cosines and sines in the sum).
\end{abstract}

Keywords: Fourier analysis, Fourier spectrum, radially periodic images, circular Fourier series, impulse rings, half-order derivatives

\section{Introduction}

Radially periodic images and their Fourier spectra occur quite frequently in optics (for example, in connection with the Fraunhofer diffraction pattern generated by circular structures). In a previous paper on the Fourier spectra of radially periodic images [1] we concentrated on circular functions whose radial periods have a symmetric profile, such as the functions shown in figures $1(a)$ and $(b)$. We showed, using Bracewell's approach [2], that any such radially periodic image can be decomposed into a circular Fourier series of circular cosine functions with radial frequencies of $f=1 / T, 2 / T, \ldots$, just as any symmetric periodic function, $p(x)$, in the one-dimensional (1D) case can be decomposed into a sum of cosines with frequencies of $f=1 / T, 2 / T, \ldots$, (the Fourier series decomposition of $p(x)$ ). We showed that this means that in the spectral domain the Fourier spectrum of such a radially periodic image consists of half-order derivative impulse rings with radii $f=n / T$ (which are the Fourier transforms of the circular cosines in the sum).

In the present paper we extend these results to the general case of radially periodic images whose radial periods do not necessarily have a symmetric profile. We show in section 2 that such a general radially periodic function can be decomposed into a circular Fourier series of circular cosine and sine functions having radial frequencies of $f=$ $1 / T, 2 / T, \ldots$ Then, we show in section 3 that, in terms of the spectral domain, the Fourier spectrum of a general radially periodic function consists of half-order derivative impulse rings with radii $f=n / T$, which are the Fourier transforms of the circular cosines and sines in the sum. The decomposition of a radially periodic image into circular cosine and sine components, rather than into a conventional series of Bessel functions, has the advantage of explicitly revealing the exact impulsive structure of the spectrum, since the $n$th term in this decomposition explicitly represents the $n$th impulse ring in the spectrum of any given radially periodic image.

\section{Image domain analysis}

A radially periodic image is a circularly symmetric image on the two-dimensional (2D) plane whose intensity profile along its radius is periodic. Its intensity profile can be visually described as the 2D surface which is obtained by revolving the positive $x$ direction of a 1D periodic function $p(x)$ about the vertical axis. A radially periodic image can therefore be expressed in terms of the polar coordinates $(r, \theta)$ as a function $g(r)$, which is periodic in $r(r>0)$. Four such functions are shown in figures $1(a)-(d)$. Note that although all of these functions are radially periodic (and hence symmetric about the origin), the two functions shown in figures $1(a)$ and $(b)$ have a radial period $T$ whose profile is symmetric with respect to the mid-period points $T / 2,3 T / 2$, etc, while the functions shown in figures $1(c)$ and $(d)$ do not share this property: in the function $f_{3}(x, y)$ of figure $1(c)$ the radial 


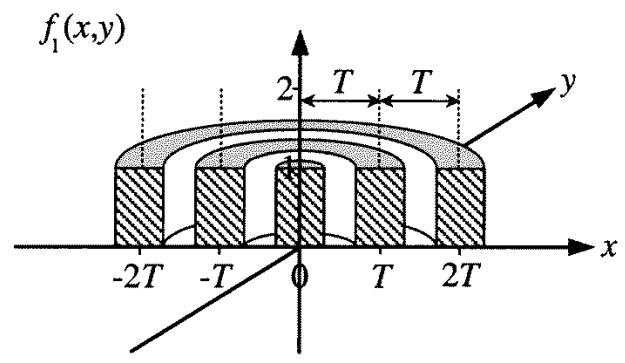

(a)

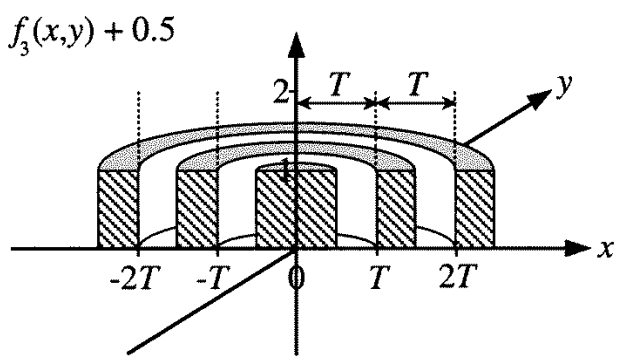

(c)

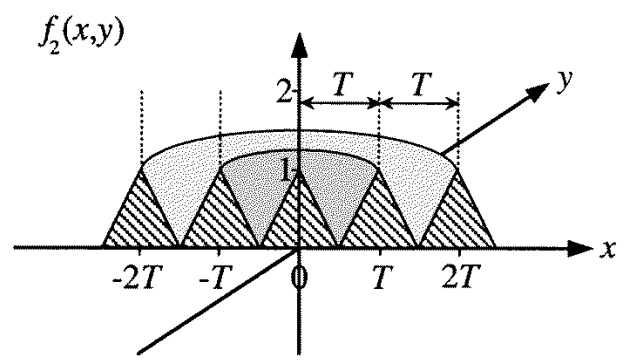

(b)

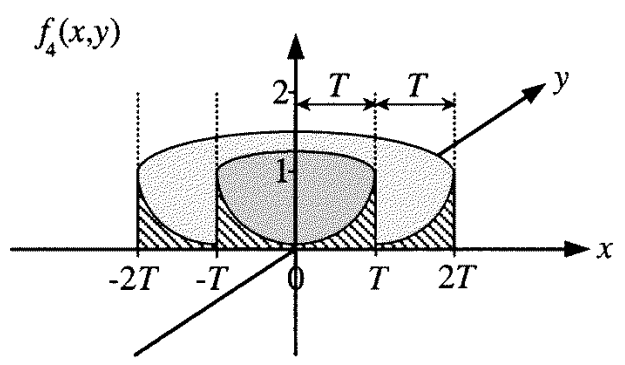

(d)

Figure 1. Four radially periodic images (gratings) on the 2D plane, all having values ranging between 0 (for black) and 1 (for white), and an identical radial period $T$. The drawings have been cut along the $x$-axis in order to clearly show their cross sections along this line (the hatched profiles). In $(a)$ and $(b)$ the profile of the radial period $T$ is symmetric with respect to its mid-period point $T / 2$; in $(c)$ the profile of the radial period $T$ is antisymmetric with respect to its mid-period point $T / 2$ (up to a vertical shift of 0.5 ); and in $(d)$ the profile of the radial period is asymmetric.

period is antisymmetric with respect to the mid-period points, and in the function $f_{4}(x, y)$ of figure $1(d)$ the radial period is asymmetric.

It was shown in [1] that just as any symmetric periodic function $p(x)$ in the 1D case can be represented by a sum of cosines with frequencies of $f=1 / T, 2 / T, \ldots$, in the $2 \mathrm{D}$ case any radially periodic image having a symmetric radial period can be decomposed into a circular Fourier series of circular cosine functions with radial frequencies of $f=1 / T, 2 / T, \ldots$ with the same Fourier coefficients $a_{n}$ as in the $1 \mathrm{D}$ case having the same waveform:

$$
g(r)=a_{0}+2 \sum_{n=1}^{\infty} a_{n} \cos \left(2 \pi \frac{n}{T} r\right) \quad(r \geqslant 0) .
$$

By a similar development it can be shown that a radially periodic image whose radial period has an antisymmetric profile with respect to the mid-period points can be decomposed into a circular Fourier series of circular sine functions, with the same coefficients $b_{n}$ as in the 1D analogue having the same waveform:

$$
g(r)=2 \sum_{n=1}^{\infty} b_{n} \sin \left(2 \pi \frac{n}{T} r\right) \quad(r \geqslant 0) .
$$

Even more generally, any arbitrary radially periodic image with a radial period $T$ can be decomposed into a circular Fourier series of circular sine and cosine functions with radial frequencies of $f=1 / T, 2 / T, \ldots$ with the same Fourier coefficients $a_{n}$ and $b_{n}$ as in the Fourier series of the 1D case having the same waveform:

$$
\begin{gathered}
g(r)=a_{0}+2 \sum_{n=1}^{\infty} a_{n} \cos \left(2 \pi \frac{n}{T} r\right)+2 \sum_{n=1}^{\infty} b_{n} \sin \left(2 \pi \frac{n}{T} r\right) \\
(r \geqslant 0)
\end{gathered}
$$

- just as any periodic function $p(x)$ in the $1 \mathrm{D}$ case can be represented by a sum of cosines and sines with frequencies of $f=1 / T, 2 / T, \ldots$.

Note, however, that in the last two cases the 1D analogue of the radially periodic grating $g(r)$ is not the periodic grating $g(x)$, but the bigrating $g(|x|)$, which is symmetric with respect to the origin. For example, the 1D analogue of $\sin (2 \pi f r)$ is $\sin (2 \pi f|x|)$; the $1 \mathrm{D}$ analogues of the radially periodic functions in figure 1 are illustrated by the hatched vertical sections.

\section{Spectral domain analysis}

It was shown in [1] that the Fourier spectrum of expression (1) consists of a series of half-order derivative impulse rings with radii $f=n / T$, which are the Fourier transforms of the circular cosines in (1).

In a similar way, the Fourier spectrum of (2) consists of a series of half-order derivative impulse rings with radii $f=n / T$, which are the Fourier transforms of the circular sines in (2). As shown in [3], the Fourier transform of a circular sine $\sin (2 \pi f r)$ is an impulse ring with radius $r=f$ which has a positive impulsive behaviour in its inner side, 


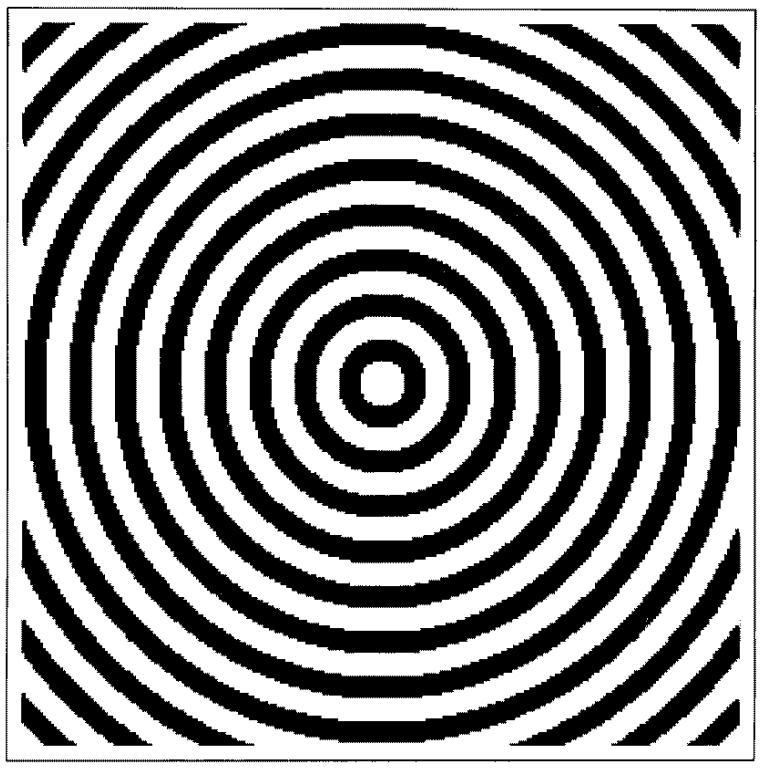

(a)

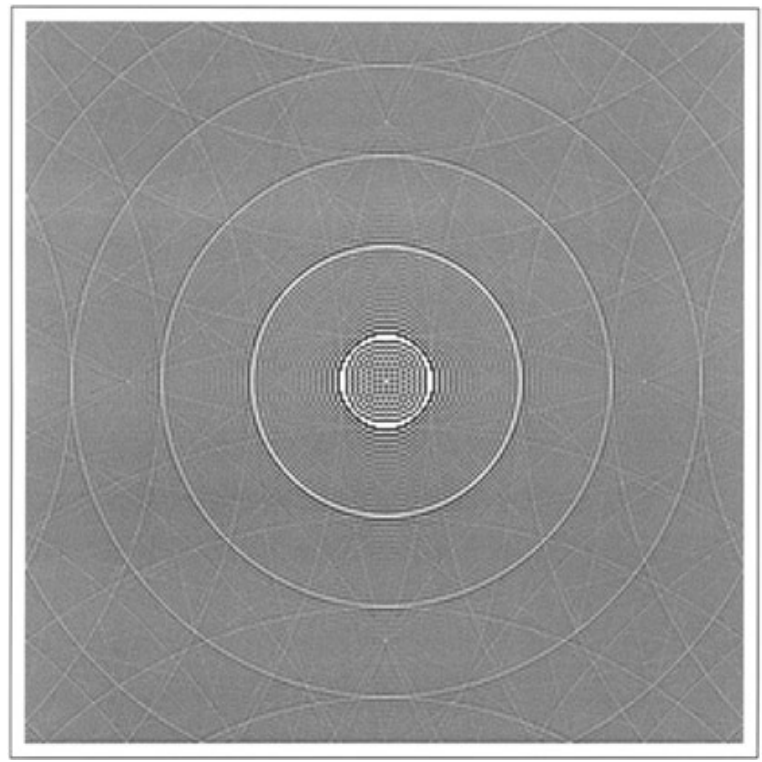

(b)

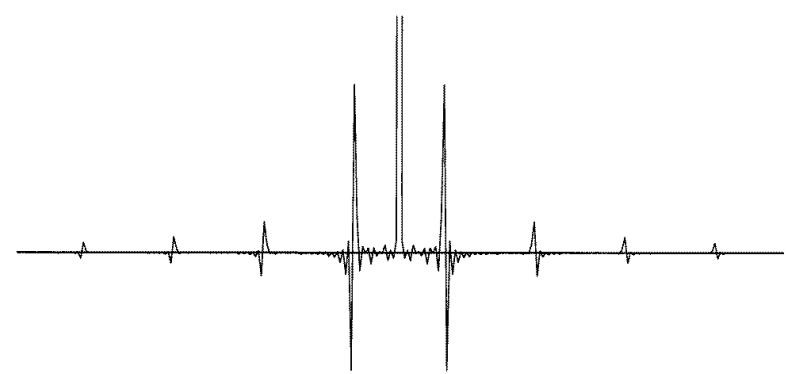

(c)

Figure 2. (a) The circular binary grating of figure 1(c), with a radial period of $T=1$ and opening of $\tau=\frac{1}{2}$. (b) Its Fourier spectrum as obtained by a 2D DFT (notice the folding-over artifacts which are due to the DFT [5]). (c) The average cross section through the origin of this DFT (averaged through all directions, $\theta=0^{\circ} \ldots 360^{\circ}$, in order to compensate for local DFT artifacts)

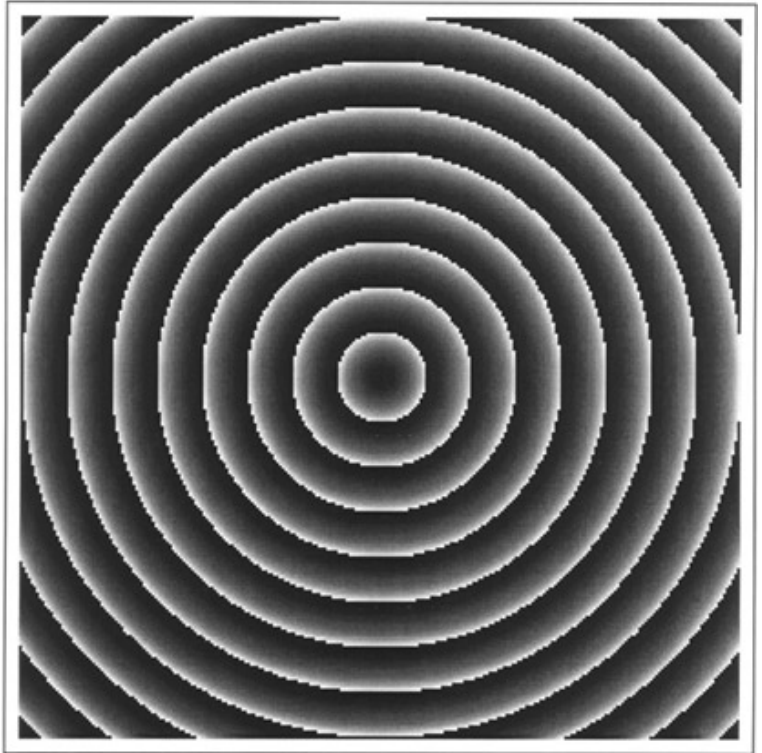

(a)

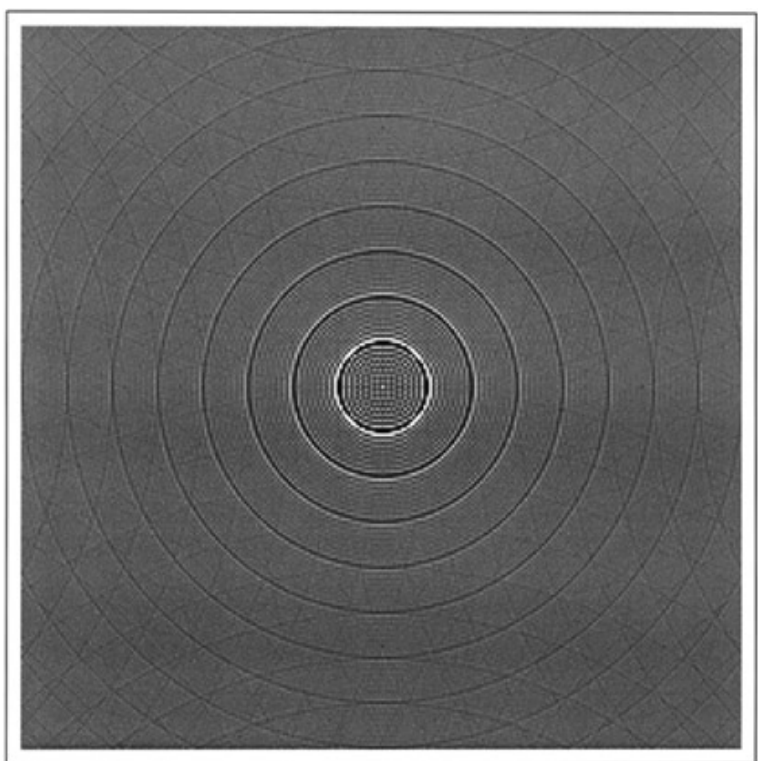

(b)

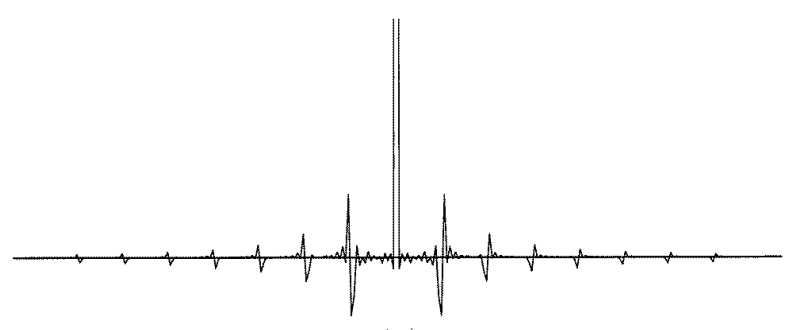

(c)

Figure 3. (a) The circular grating of figure $1(d)$, with an asymmetric radial period of $T=1$. (b) Its Fourier spectrum as obtained by a 2D DFT (notice the folding-over artifacts which are due to the DFT [5]). (c) The average cross section through the origin of this DFT (averaged through all directions,

$\theta=0^{\circ} \ldots 360^{\circ}$, in order to compensate for local DFT artifacts). 
and a negative impulsive behaviour in its outer side, which trails off outwards as a negative, continuous wake. This is, in fact, the inside-out inversion of the impulse ring in the Fourier transform of the circular cosine, $\cos (2 \pi f r)$. This can be clearly seen from the explicit expressions of these Fourier (or rather Hankel $\dagger$ ) transforms:

$$
\begin{aligned}
& \cos (2 \pi f r) \stackrel{\mathcal{H}}{\longleftrightarrow} \frac{f}{\sqrt{\pi}} \frac{1}{(f+q)^{3 / 2}} \delta^{(1 / 2)}(f-q) \\
& \sin (2 \pi f r) \stackrel{\mathcal{H}}{\longleftrightarrow} \frac{f}{\sqrt{\pi}} \frac{1}{(f+q)^{3 / 2}} \delta^{(1 / 2)}(q-f) .
\end{aligned}
$$

It follows, therefore, that the Fourier spectrum of expression (3) consists of a series of half-order derivative impulse rings with radii $f=n / T$, which are weighted sums of the Fourier transforms of the circular cosines and sines $a_{n} \cos \left(2 \pi \frac{n}{T} r\right)+$ $b_{n} \sin \left(2 \pi \frac{n}{T} r\right)$ in (3). These impulse rings therefore have continuous wakes trailing both inwards (the contribution of the circular cosine) and outwards (the contribution of the circular sine).

These results are indeed confirmed by $2 \mathrm{D}$ discrete Fourier transform (DFT), as shown in the examples in figures 2 and 3 . Figure 2 shows the radially periodic function of figure 1(c), whose radial period has an antisymmetric profile, and its spectrum as obtained by 2D DFT. As we can see, the spectrum consists of a series of impulse rings having negative continuous wakes which trail off outwards; these are the Fourier transforms of the circular sines in the series development (2), whose amplitudes are weighted by the Fourier series coefficients $b_{n}$. Note that all the even rings (the rings whose radii are $r=n / T$ with even $n$ ) have a zero amplitude and do not appear in the spectrum; this is due to the opening ratio $\tau / T=\frac{1}{2}$ of the square wave, since the Fourier coefficients in the series development of a square wave are given by $b_{n}=(1 / n \pi) \sin (n \pi \tau / T)$.

Figure 3 shows the radially periodic function of figure $1(d)$, whose radial period has an asymmetric profile, and its spectrum as obtained by 2D DFT. As we can see, the spectrum consists of a series of impulse rings having continuous wakes which trail off both inwards and outwards; these are the Fourier transforms of the circular cosines and sines in the series development (3), $a_{n} \cos \left(2 \pi \frac{n}{T} r\right)+$ $b_{n} \sin \left(2 \pi \frac{n}{T} r\right)$, whose amplitudes are weighted by the Fourier series coefficients $a_{n}$ and $b_{n}$.

The decomposition of a radially periodic image into circular cosine and sine components offers a significant advantage over other possible decompositions (such as the development into a series of Bessel functions) in that it explicitly reveals the exact impulse ring structure of the spectrum and the special properties of each of these rings: in the proposed circular Fourier decomposition, the $n$th term explicitly represents the $n$th impulse ring in the spectrum of the radially periodic image.

$\dagger$ The Hankel transform is an equivalent way to express the 2D Fourier transform of a circularly symmetric function, based on its radius; see, for example, [4]. Both terms can be used interchangeably, depending on the coordinate system implied, polar or Cartesian.

\section{The case of circular images with a finite number of periods}

Just as in the 1D case, an image which only contains a finite number of periods is not a periodic image; it is, in fact, the product of an initial radially periodic image, having infinitely many periods, with a low-pass filter, $\operatorname{rect}(r / d)$, having the appropriate cut-off frequency. This means that the spectrum of such an image is no longer impulsive, since the multiplication of the initial radially periodic image with $\operatorname{rect}(r / d)$ in the image domain implies that in the spectral domain the initial impulsive spectrum is convolved with the continuous spectrum of $\operatorname{rect}(r / d)$.

For example, in the case of a circular image $g_{9}(r)$ that consists of nine concentric slits, it was shown in [2, pp 817] that the spectrum is composed of concentric ringlobes, where the profile of each ringlobe can be approximated in terms of a half-order derivative of the sinc function [2, equation (12)]. This spectrum is clearly continuous and not impulsive. However, when the number of periods in the image-domain function tends to infinity, each of the continuous ringlobes in the spectrum again becomes a halforder derivative impulse ring [2, pp 89-90]. This result is not specific to the present example, and it holds for any circular image with a finite number of periods: since when the number of periods tends to infinity, the image-domain multiplication with the filter $\operatorname{rect}(r / d)$ turns into a multiplication with the identical 1-function, so that in the spectral domain the initial, impulsive spectrum is now convolved with a simple impulse (the Fourier transform of the identical 1-function), meaning that it remains unchanged.

It is interesting to note, however, that when we consider the power spectrum (i.e. the squared spectrum) of an image with a finite number of periods (for example, the Fraunhofer diffraction pattern of the image), then the centres of the observed ringlobes may seem to be located slightly away from their expected radii of $f=n / T$. This was observed in [6] in the diffraction pattern of the finite circular image $g_{9}(r)$. This phenomenon is due to the fact that the function that describes the profile of the ringlobe in the unsquared spectrum (in our case, the half-order derivative of the sinc function) is not centred about the zero-crossing between its positive and negative peaks (see figure 4 , top). When the power spectrum of our finite circular image is considered, one often takes as the 'centre' of each ringlobe the central zerovalued ring which corresponds to the zero crossing between the positive and negative peaks in the unsquared spectrum. However, as we have just seen, this zero-valued ring is not the real centre of the ringlobe, and is therefore located slightly away from the radius $f=n / T$, as clearly shown in figure 4. This gives the impression that the ringlobe radii that are measured in the power spectrum (or the diffraction pattern) are not exactly $f=n / T$, as mentioned in [6]. However, as can be seen in the unsquared spectrum (and in equation (12) of [2]), each of the ringlobes (the sinc ${ }^{(1 / 2)}$ functions) is indeed centred exactly at $f=n / T$, although its mathematical centre does not coincide with the zero-crossing point.

It should be stressed that this seeming discrepancy only happens because the image in question consists of a finite 

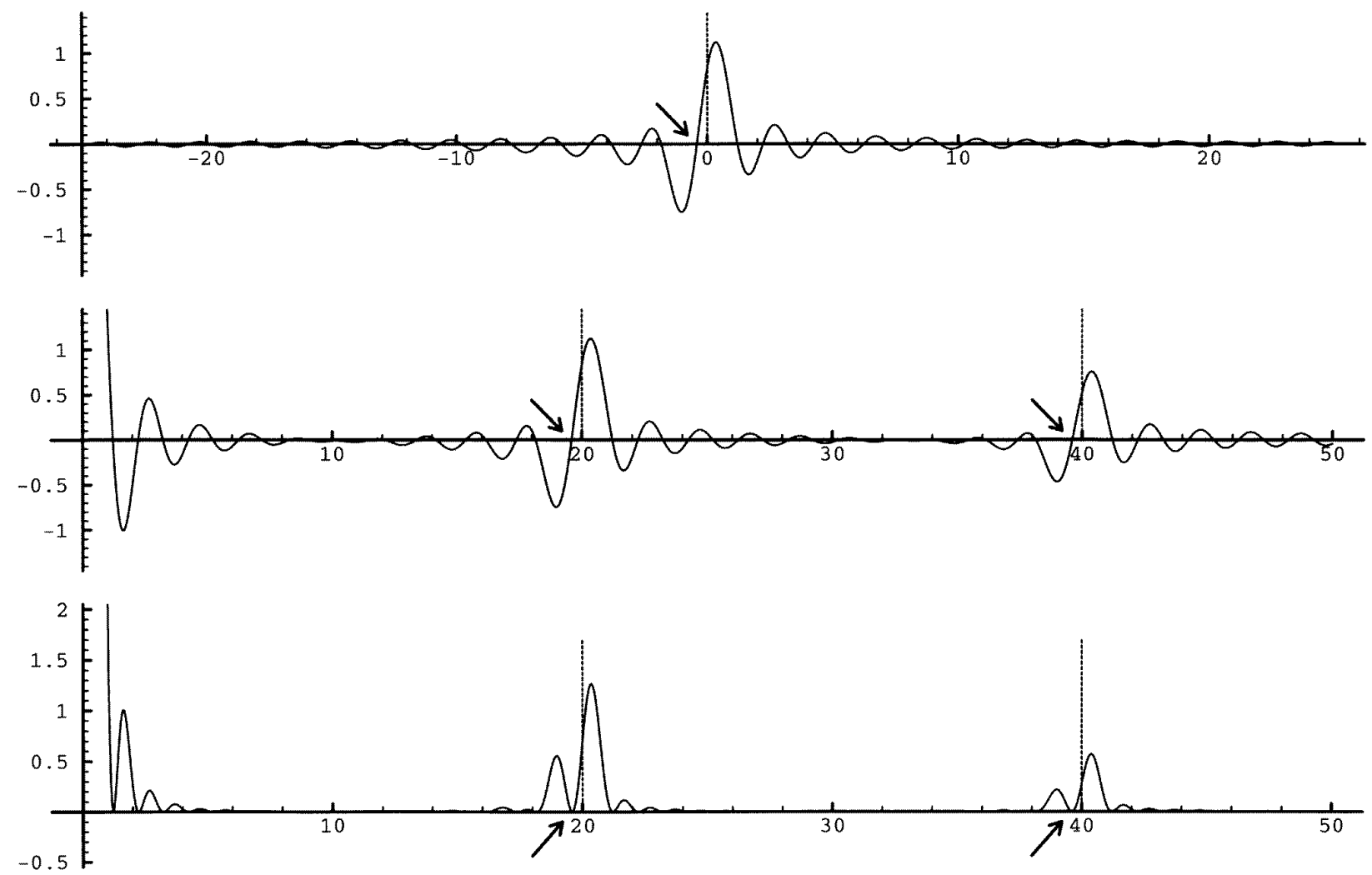

Figure 4. Top: the function $\operatorname{sinc}^{(1 / 2)}(-x)$, clearly showing the off-centre location of the central zero-crossing point (the reasons for the sign inversion are explained in [1] pages 822 and 825). Centre: a cross section through the first two ringlobes in the spectrum of a circular image with a finite number of radial periods having a symmetric profile. Bottom: a cross section through the first two ringlobes in the power spectrum of the same image. Dotted lines indicate the mathematical centre of the function $\operatorname{sinc}^{(1 / 2)}(-x)$ and of each of the ringlobes, while the arrows indicate the central zero-crossing point in each case. As clearly shown, although the mathematical centres of the ringlobes are indeed equally spaced, if one considers the central zero-crossing point as the centre of each ringlobe in the power spectrum (as this is the most easily detected reference point in the middle of the ringlobe), the radius of the second-order ringlobe does not seem to be exactly twice the radius of the first-order ringlobe.

number of periods, and is therefore not a radially periodic image. Had it been a radially periodic image, with infinitely many periods, each of the ringlobes in its spectrum would have reduced into a half-order derivative impulse ring, as explained earlier, whose positive and negative peaks as well as the zero crossing between them coincide precisely at the radius $f=n / T$.

\section{Summary}

It has been shown that a general radially periodic image $g(r)$, whose radial period does not necessarily have a symmetric profile, can be decomposed into a Fourier series of circular cosine and sine functions with radial frequencies of $f=$ $1 / T, 2 / T, \ldots$ In terms of the spectral domain, the spectrum of the radially periodic function $g(r)$ consists of a DC impulse plus a series of half-order derivative impulse rings with radii of $f=1 / T, 2 / T, \ldots$, each of which is the spectral representation of the term $a_{n} \cos \left(2 \pi \frac{n}{T} r\right)+b_{n} \sin \left(2 \pi \frac{n}{T} r\right)$ in the image-domain decomposition. The particular wakes of these impulse rings, which may trail off both inwards and outwards, are simply a weighted sum of the wakes in the Fourier spectra of the corresponding circular cosine and sine functions. Finally, these results are also extended to the case of circular images with a finite number of periods, and a particular artifact which may occur in such cases is explained and illustrated.

It should be noted that although in this paper we have only discussed real-valued images $g(r)$, our results may be also extended, by using elementary Fourier properties [7], to pure imaginary-valued radially periodic images $\mathrm{i} g(r)$ and hence also to any complex-valued radially periodic images $g_{1}(r)+\mathrm{i} g_{2}(r)\left(g_{1}(r)\right.$ and $g_{2}(r)$ being real-valued radially periodic images with the same radial period).

\section{References}

[1] Amidror I 1997 J. Opt. Soc. Am. A 14 816-26

[2] Bracewell R N and Thompson A R 1973 Astrophys. J. 182 77-94

[3] Amidror I 1998 Opt. Commun. 149 127-34

[4] Bracewell R N 1986 The Fourier Transform and its Applications 2nd edn (Reading, MA: McGraw-Hill) p 244

[5] Brigham E O 1988 The Fast Fourier Transform and its Applications (Princeton, NJ: Prentice-Hall) pp 172-3

[6] Tichenor D A and Bracewell R N 1973 J. Opt. Soc. Am. 63 $1620-2$

[7] Bracewell R N 1986 The Fourier Transform and its Applications (New York: McGraw-Hill) pp 16-17 